\title{
POLYA-VINOGRADOV INEQUALITY FOR POLYNOMIAL CHARACTER SUMS OVER FINITE FIELDS
}

\author{
ZHIYONG ZHENG AND ZiWEI HONG
}

\begin{abstract}
A version of Polya-Vinogradov inequality in function fields appeared in [1, 2, 3, 9] recently. In this paper, we show some new bounds for polynomial character sums by making use of polynomial Gauss sums (see [4, 12]) and a formula from L. Carlitz (see [5]) on exponential sums over function fields. The method is elementary. It is worth mentioning that the proofs given in this paper do not depend on the well-known result form A. Weil on L-function associated to algebraic curves over finite fields.
\end{abstract}

Mathematics subject classification (2020): 11T55, 11T24.

Keywords and phrases: Polya-Vinogradov inequality, Gauss sums, character sums, function fields.

\section{REFERENCES}

[1] J. C. Andrade, J. P. Keating, Conjectures for the integral moments and ratio of L-functions over function fields, J. of Number Theory., 142 (2014), 102-148, doi:10.1016/j.jnt.2014.02.019

[2] J. C. AndRADE, Rudnick and Soundararajan's theorem for function fields, Finite Fields Appl., 37(2016), 331-327, https://doi.org/10.1016/j.ffa.2015.10.007.

[3] S. Bae And And P. L. Kang, Gauss Sums on $\mathbb{F}_{q}[T]$, J. Chungchong Math. Soc., 23 (2010), $757-$ 771, http://www.ccms.or.kr/data/pdf paper/jcms23_4/23_4_757.pdf.

[4] L. CARlitz, The Singular series for Sums of Squares of Polynomials, Duke Math. J., 14 (1947), 1105-1120, doi:10.1215/S0012-7094-47-01484-1.

[5] L. Carlitz, Diophantine Approximation in Field of Characteristic p, Trans. Amer. Math. Soc, 72 (1952), 187-208, doi:10.1090/S0002-9947-1952-0048503-0.

[6] O. W. EfFinger And D. R. HAYes, Additive Number Theory of Polynomial over Finite Fields, Clarendon Press, Oxford, 1991.

[7] D. Faifman, Z. Rudnick, Statistics of the Zeros of Zeta Function in Families of the Hyperlliptic Curve over a Finite Field, Compos. Math. 146 (2010), 81-101, doi:10.1112/S0010437X09004308.

[8] D. R. HaYes, A Polynomial Generalized Gauss Sums, J. Reine Angew. Math., 222 (1966), 113-119.

[9] C. Hsu, Estimates for Coefficients of L-Functions for Function Fields, Finite Fields Appl., 5 (1999), 76-83, doi:10.1006/ffta.1998.0234.

[10] M. Rosen, Number Theory in Function Fields, Springer-Verlag, New York Berlin Heidelberg, 2001.

[11] M. H. Taibleson, Fourier Analysis on Local Fields, Princeton University Press and University of Tokyo Press, 1975.

[12] ZhiYong Zheng, Davenport-Hasses Theorem for Polynomial Gauss Sums over Finite Fields, J. Number Theory, 180 (2017), 460-473, https://doi.org/10.1016/j.jnt.2017.04.005.

[13] ZhiYong Zheng, On the polynomial Ramanujan Sums over Finite Fields, Ramanujan J., 46 (2018), 863-898. 